\title{
Structural dimensions depending on light intensity in a 3D printing method that utilizes in situ light as a guide
}

\author{
Jongkyeong Lim', Sangmin Lee ${ }^{2^{*}}$ (1) and Joonwon Kim*
}

\begin{abstract}
Conventional 3D printing methods require the addition of a supporting layer in order to accurately and reliably fabricate the desired final product. However, the use of supporting material is not economically viable, and during the process of removing the supporting material, the shape or the properties of the final product may be distorted. In our previous work, we proposed and demonstrated the concept of a new 3D printing method that utilizes the in situ light as a guide for the fabrication of freestanding overhanging structures without the need for supporting material. In this study, the influence of the light intensity on the diameter of the structure and the thickness of the layer produced per droplet is analyzed in order to identify the geometric range of structures that can be fabricated by the new 3D printing method. As the intensity of the light increased, the diameter of the structure also increased and the thickness of the layer per droplet decreased. This result is determined by a combination of factors; (1) the rebound motion of the photocurable droplet and (2) the surface area of the structure that needs to be covered.
\end{abstract}

Keywords: 3D printing, Light guide, Light intensity, Layer thickness, Structure diameter

\section{Introduction}

Conventional 3D printing methods such as polyjet [1], stereolithography [2], fused deposition modeling [3-5], and selective laser sintering [6] require the addition of a supporting layer (generally known as the support) in order to accurately and reliably fabricate the final product desired. This layer is essential for the fabrication of structurally unstable overhanging or serpentine structures [7]. However, the use of supporting material is not economically viable, as it requires the consumption of secondary materials in addition to those necessary for the fabrication of the final product, increasing the complexity of the production process. Removal of the support is carried out via physical or chemical methods following

\footnotetext{
*Correspondence: thinking@deu.ac.kr; joonwon@postech.ac.kr ${ }^{1}$ Department of Mechanical Engineering, Pohang University of Science and Technology (POSTECH), 77 Cheongam-Ro, Nam-Gu, Pohang, Gyeongbuk 37673, Korea

${ }^{2}$ Division of Mechanical, Automotive and Robot Component Engineering, Dong-Eui University, 176 Eomgwang-Ro, Busanjin-Gu, Busan 47340, Korea
}

fabrication, and the shape or properties of the final product can be distorted during removal. Physical removal may lead to damage on the surface of a product, and remnants of the material used for support may remain on the product [7]. Chemical removal of the support can also contaminate the final product or cause structural deformation [8]. In our previous work $[9,10]$, we proposed and demonstrated the concept of a new 3D printing method that utilizes in situ light as a guide in order to fabricate freestanding overhanging structures without the need for support. However, the effects of various factors, such as the properties of the photocurable droplets, the printing conditions, and the environment, on the dimensions and geometry of the structure fabricated through the new 3D printing method have not been sufficiently analyzed. In particular, the curing characteristics and behavior of the photocurable droplets are the main factors that determine the possible dimensions of the structure $[11,12]$.

This paper analyzes the influence of light intensity on the diameter of a structure and the thickness of the layer produced per droplet in order to identify the geometric range 
of structures that can be fabricated using the new 3D printing method. The deposition of photocurable droplets (from which changes occur in the height of the structure after deposition) was observed with a high speed camera while varying the intensity of the light within a range between 50 and $300 \mathrm{~mW} / \mathrm{cm}^{2}$. Experimental analysis was then carried out to discern the effects caused by the motion of the photocurable droplet, such as rebound or spreading, on the diameter of the structure and the thickness of the layer produced per droplet.

\section{Materials and methods \\ Materials}

All materials were purchased from Sigma-Aldrich unless otherwise noted. The photocurable prepolymer was prepared by mixing $90 \% \mathrm{v} / \mathrm{v}$ poly (ethylene)glycol diacrylate (PEGDA, $575 \mathrm{Da}$ ) with $10 \% \mathrm{v} / \mathrm{v}$ deionized water, which was followed by the addition of $0.1 \mathrm{mM}$ eosin $\mathrm{Y}, 1.5 \% \mathrm{w} / \mathrm{v}$ triethanolamine, and $1 \% \mathrm{w} / \mathrm{v}$ 1-vinyl-2 pyrrolidinone. The photocurable prepolymer had a dynamic viscosity of $45-60 \mathrm{mPa}$, with an increasing shear rate of $0-1000 / \mathrm{s}$ at $25^{\circ} \mathrm{C}$ and a surface tension of $\approx 38 \mathrm{mN} / \mathrm{m}$ at $23^{\circ} \mathrm{C}$.

\section{Experimental setup}

The 3D printing system consisted of a pneumatic printing system [13], a $532 \mathrm{~nm}$ laser (CNI), mirrors (Thorlabs), and a glass substrate. This pneumatic printing system allows for the continual ejection of high-viscosity photocurable prepolymer in the form of single droplets (i.e., the photocurable droplets). The intensity of the light can be adjusted within the range $0-3 \mathrm{~W} / \mathrm{cm}^{2}$. The mirrors were placed on angle adjustable mounts to ensure that the light emitted from the laser was fixed at the desired point on the glass substrate. The glass substrate both supported the structure and transmitted the light.

\section{Analysis of the droplet behavior}

The process of deposition was observed at 30,000 frames per second (FPS) using a high-speed camera (Fastcam mini AX100, Photron), from the point at which the photocurable droplet collided with the structure to the completion of curing. The images produced by high-speed camera were analyzed using MATLAB software (MathWorks). The dynamic viscosity and the surface tension of the photocurable prepolymer were then measured using a rheometer (Discover HR-2, TA Instruments) and a surface tension measurement system (SmartDrop, Femtofab), respectively.

\section{Results and discussion}

Principle of the 3D printing method utilizing an in situ light as a guide

The 3D printing method that uses in situ light as a guide for printing is inspired by the transmission of light in optical fibers (Fig. 1), in which light is transmitted through the core of the fiber via total reflection, which occurs because of the difference between the refractive indexes of the core and the cladding. Total reflection indicates that none of the light is transmitted through a surface, but that $100 \%$ of light is reflected. In order for total reflection to occur, the light is irradiated at an angle of incidence greater than the critical angle, from a medium with a higher refractive index to a medium with a lower refractive index. Therefore, to use the in situ light guide in air (which has a refractive index of $\approx 1$ ), the refractive index of the material used should be greater than 1 . The light emitted from the laser is reflected via the mirrors and fixed onto the desired point on the glass substrate. A photocurable droplet is then ejected through the pneumatic printing system to the desired point on the glass substrate and is polymerized directly by the light, forming an initial structure. This initial structure guides the light until the structure is complete, and the ejected photocurable droplets are then polymerized by both the guiding light and the light transmitted during the process of deposition.

\section{Structural dimensions depend on the intensity of the light} Light intensity is one of the important factors (e.g., droplet volume, velocity, viscosity, and surface tension) that determines the dimensions of a $3 \mathrm{D}$ printed structure, such as the diameter or the thickness of the layer produced per droplet when using in situ light as a guide. The conditions necessary to form stable structures while avoiding clogging in the nozzle were determined via experiment, as reported previously [9, 10]; this involves using a standoff distance of $2 \mathrm{~cm}$.

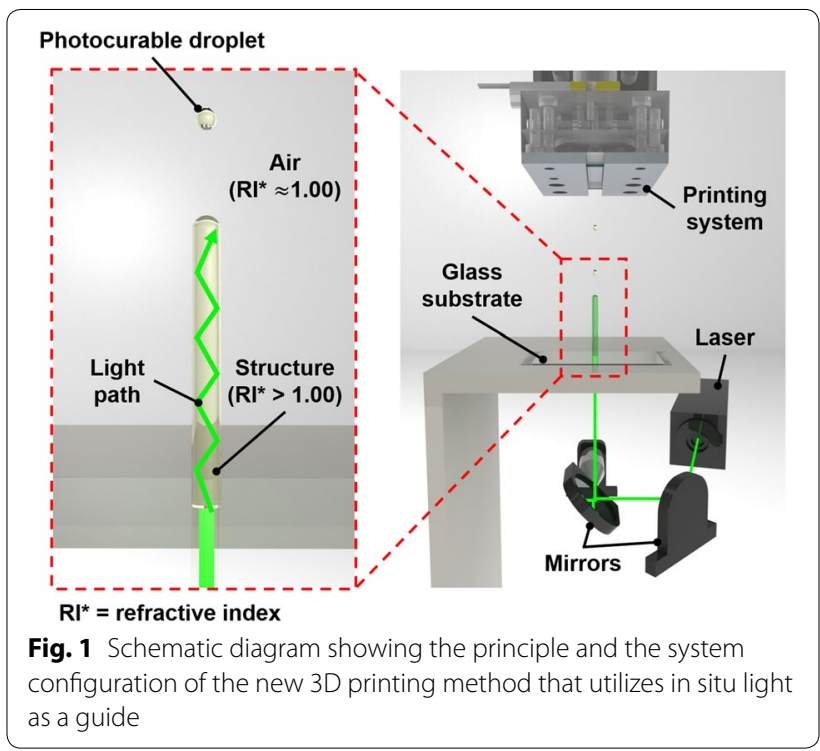


The volume and velocity of the droplets were fixed at $\approx 70 \mathrm{~nL}$ and $\approx 1.1 \mathrm{~m} / \mathrm{s}$, respectively. The diameter of the structure and the thickness of the layer produced per droplet were investigated under different light conditions by increasing the light intensity to $50,75,100$, 200 , and $300 \mathrm{~mW} / \mathrm{cm}^{2}$. The parameters of the light intensity were determined experimentally, taking into account the need for consistent ejection of the droplets through the nozzle and for the formation of the structure through light curing of the droplets. At light intensities greater than $\approx 300 \mathrm{~mW} / \mathrm{cm}^{2}$, droplets were not ejected due to clogging in the nozzle, and at less than $\approx 25 \mathrm{~mW} / \mathrm{cm}^{2}$, the structure could not be formed due to a low curing rate and a limited light exposure. In order to fabricate a certain height or more (i.e., $\approx 5 \mathrm{~cm}$ ), the minimum light intensity was determined to be $\approx 50 \mathrm{~mW} / \mathrm{cm}^{2}$, taking into consideration that the attenuation of light in a fiber made of $90 \%$ PEGDA is $\approx 0.30 \mathrm{~dB} / \mathrm{cm}$ [14]. It is thought that the curing rate of photocurable droplets becomes faster as the intensity of the light is increased, reducing the time it takes for the photocurable droplet to spread after deposition. It is therefore expected that the diameter of the structure will decrease and the thickness of the layer per droplet will increase as the intensity of the light increases. However, experimentation indicated that as the intensity of the light increased, the diameter of the structure increased from $\approx 0.9$ to $\approx 1.2 \mathrm{~mm}$, and the thickness of the layer produced per droplet decreased from $\approx 100$ to $\approx 60 \mu \mathrm{m}$ (Fig. 2). In order to analyze the cause of these results, the deposition of a photocurable droplet was observed with a high speed camera while the light intensity was increased.

\section{The process of deposition of the photocurable droplet on the structure}

Changes occurring in the height of the structure were analyzed in order to verify the motion of the photocurable droplets during deposition. Different patterns were observed in terms of rebound and the height of the structure (Fig. 3a, b), which were associated with the intensity of the light. Irrespective of light intensity, the deposition of a photocurable droplet can be divided into two domains; (1) an unstable domain (before $5 \mathrm{~ms}$ ), immediately following deposition, during which several different types of motion are observed in the droplets, and (2) a stable domain (after $5 \mathrm{~ms}$ ), during which a structure is formed as polymerization proceeds (Fig. 3c). Differences were observed in the motion of the photocurable droplets in the unstable domain with an increase in the light intensity. At a light intensity of $50 \mathrm{~mW} / \mathrm{cm}^{2}$, the photocurable droplet underwent a clear rebound motion after colliding with the structure, but this motion decreased gradually as the light intensity was increased, becoming indistinguishable at $300 \mathrm{~mW} / \mathrm{cm}^{2}$. This is due to the difference in the polymerization of the photocurable droplet over the same period of time, which is caused by an increase in the curing rate of the photocurable droplet as the light intensity increases. The viscosity of the droplet increases rapidly as polymerization proceeds [15] and the density increases due to the reduction in volume [16], but the surface tension does not change significantly [17]. Therefore, the faster curing rate that is due to the higher light intensity causes a decrease in the flowability because of the increase in the viscosity of the photocurable droplet, which in turn changes the motion of the rebound in the unstable domain. Following the unstable period, the photocurable droplet spreads slowly over the surface of the structure and is gradually

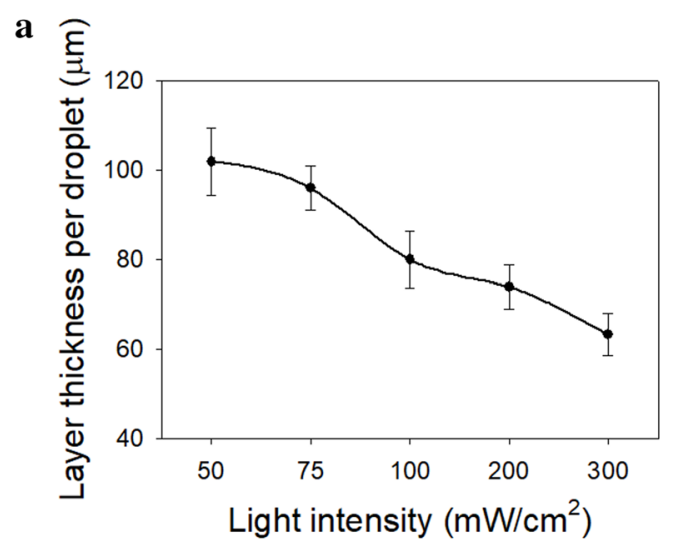

b

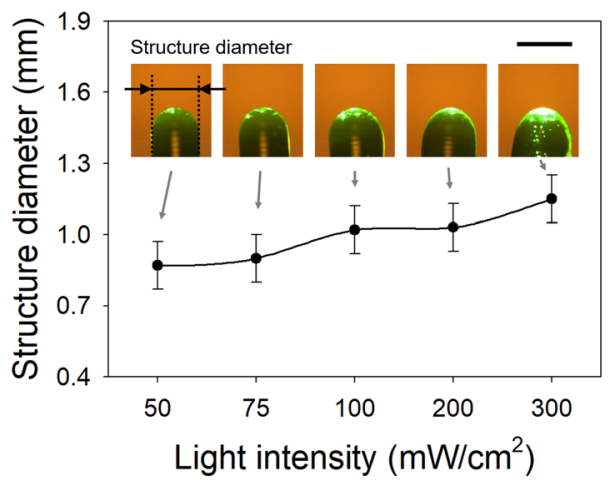

Fig. 2 Structural dimensions depending on light intensities $\left(\mathrm{mW} / \mathrm{cm}^{2}\right)$. a Layer thickness per droplet $(\mu \mathrm{m})$. b Structure diameter $(\mathrm{mm})$. The scale bar is $1 \mathrm{~mm}$ 


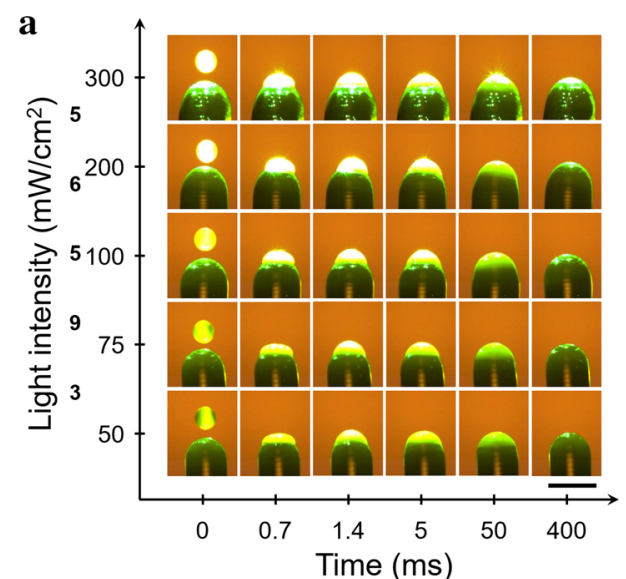

c

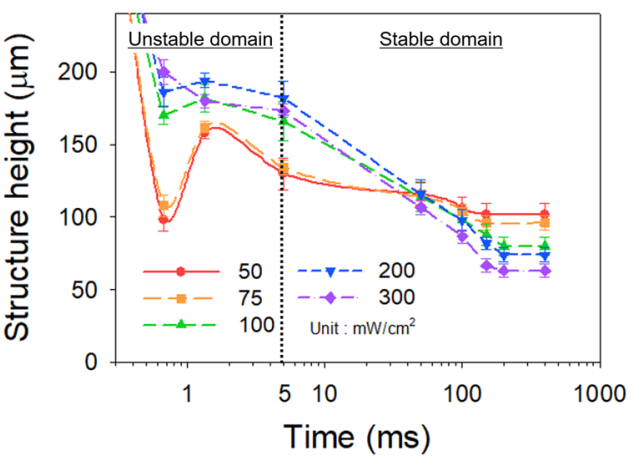

b

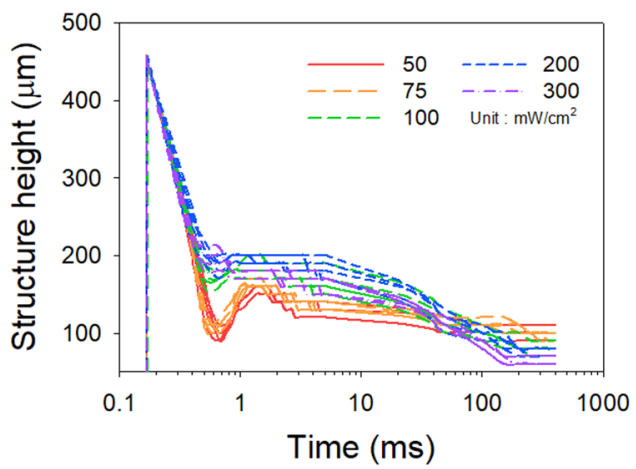

d

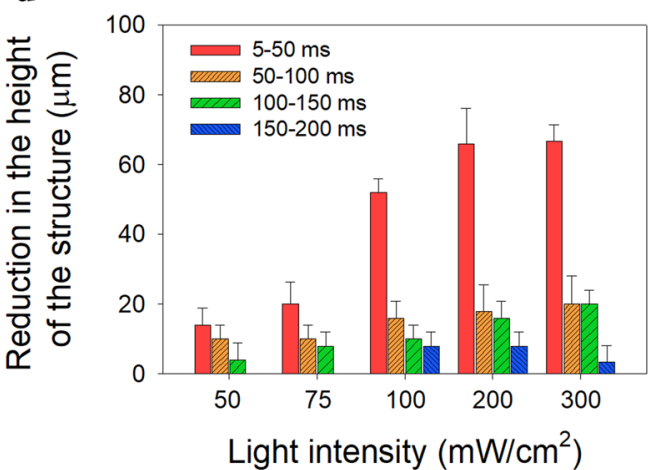

Fig. 3 The process of deposition of the photocurable droplet on the structure. $\mathbf{a}$ High speed images. $\mathbf{b}, \mathbf{c}$ Changes occurring in the height of the structure. $\mathbf{d}$ The reduction in the height of the structure in accordance with the time period. The scale bar is $1 \mathrm{~mm}$

polymerized until the final completion. This period is called the stable domain, and it is divided into four periods of time $(5-50 \mathrm{~ms}, 50-100 \mathrm{~ms}, 100-150 \mathrm{~ms}$, and 150-200 ms) in order to analyze the reduction in height in accordance with the time period. As the intensity of the light is increased in the stable domain, the reduction in the height of the structure also increases over each period of time (Fig. 3d). This means that as the light intensity increases, there is a decrease in the thickness of the layer per droplet. On the other hand, the diameter of the structure increases with increasing light intensity, as can be seen in Fig. 2a. The greater the diameter, the larger the surface area over which the droplet can be spread; this means that the droplet spreads out slowly until the surface is covered. The reduction in the thickness of a layer is therefore inversely proportional to the area over which a droplet spreads. In other words, the thickness of the layer per droplet appears to be more affected by the diameter of the structure than the flowability of the droplet in the stable domain. The rebound motion immediately influences the height of the structure in the unstable domain, and the diameter of the structure affects the reduction in height of the structure in the stable domain, determining the thickness of layer produced per droplet.

It has been confirmed by previous studies that the diameter of a structure affects the reduction in the height of a structure in the stable domain. However, it is not yet clear why the diameter of a structure increases with light intensity. It is speculated that the rebound motion that occurs after the droplet collides with the structure affects the diameter of the initial structure, but further analysis of the process by which the initial structure is formed in terms of light intensity is required to clarify this.

\section{Conclusion}

In this study, the dimensions of a structure such as the diameter and the thickness of the layer produced per droplet were measured in a new $3 \mathrm{D}$ printing process in which in situ light is used as a guide while varying the light intensity within the range $50-300 \mathrm{~mW} / \mathrm{cm}^{2}$, and the effects that the motion of the photocurable droplet such as rebound and spreading have on the dimensions of a structure were experimentally analyzed. In the new 3D printing method, as the intensity of the light increased 
the diameter of the structure also increased and the thickness of layer produced per droplet was observed to decrease. The process of dispersion was divided into an unstable domain, in which various motions are observed immediately after collision, and a stable domain in which structures are formed via gradual polymerization. In the unstable domain, greater light intensity led to a decrease in the rebound of the droplets. This is because the increase in the curing rate of the photocurable droplets caused by the high light intensity led to a decrease in flowability that was due to an increase in the viscosity of the photocurable droplets. As the light intensity is increased in the stable domain, the reduction in the height of the structure also increases over each period of time. This is because the greater the diameter of a structure, the larger the surface area over which the droplets can spread, and the droplets spread until they cover the surface. The thickness of a layer therefore decreases by the same volume as the area to be covered by a droplet. This means that the size of the surface of the structure was more affected than the flowability of the droplets in the stable domain.

As a result, the higher the light intensity, the thinner the thickness of the layer produced per droplet. This phenomenon is determined by the combined factors of the low rebound that is due to the reduced flowability of the photocurable droplet at high light intensities and the wide surface area of the structure to be covered.

\section{Acknowledgements}

Not applicable.

\section{Authors' contributions}

LJ performed the experiments, analyzed the data and wrote the manuscript. SM and KJ supervised the research and reviewed the manuscript. All authors read and approved the final manuscript.

\section{Funding}

This research was supported by a grant of the Korea Health Technology R\&D Project through the Korea Health Industry Development Institute (KHIDI), funded by the Ministry of Health and Welfare, Republic of Korea (Grant No. HI15C0001) and the National Research Foundation of Korea (NRF) funded by the Ministry of Science and ICT (MSIT) (Grant No. NRF-2017R1A2B4003328 and 2017R1C1B5076710).

\section{Availability of data and materials}

The datasets supporting the conclusions of this article are included within the article.

\section{Competing interests}

The authors declare that they have no competing interests.
References

1. Ibrahim D, Broilo TL, Heitz C, De Oliveira MG, De Oliveira HW, Nobre SMW, Dos Santos Filho J H G, Silva DN (2009) Dimensional error of selective laser sintering, three-dimensional printing and PolyJet models in the reproduction of mandibular anatomy. J Craniomaxillofac Surg 37:167-173

2. Melchels FPW, Feijen J, Grijpma DW (2010) A review on stereolithography and its applications in biomedical engineering. Biomaterials 31:6121-6130

3. Lee Y, Han J, Choi B, Yoon J, Park J, Kim Y, Lee J, Kim DH, Kim DM, Lim M, Kang M-H, Kim S, Choi S-J (2018) Three-dimensionally printed microelectromechanical switches. ACS Appl Mater Interfaces 10:15841-15846

4. Lim J, Kim A-R, Kim S, Lee S, Yoo D, Park J, Kim J (2019) A new dip coating method using supporting liquid for forming uniformly thick layers on serpentine 3D substrates. Adv Mater Interfaces 6:1901485

5. Espalin D, Muse DW, MacDonald E, Wicker RB (2014) 3D printing multifunctionality: structures with electronics. Int J Adv Manuf Technol 72:963-978

6. Appleyard D (2015) Powering up on powder technology. Met Powder Rep 70:285-289

7. Hu K, Jin S, Wang CCL (2015) Support slimming for single material based additive manufacturing. Comput Aided Des 65:1-10

8. Hinton TJ, Jallerat Q, Palchesko RN, Park JH, Grodzicki MS, Shue H-J, Ramadan MH, Hudson AR, Feinberg AW (2015) Three-dimensional printing of complex biological structures by freeform reversible embedding of suspended hydrogels. Sci Adv 1:e1500758

9. Lim J, Kim YK, Won D-J, Choi IH, Lee S, Kim J (2019) 3D printing of freestanding overhanging structures utilizing an in situ light guide. Adv Mater Technol 4:1900118

10. Lim J, Lee S, Won D-J, Choi I H, Kim J (2019) Simple manufacturing approach for 3D overhanging structure of hydrogel with in situ lightguiding mechanism. In: Abstracts of the 31 th International conference on micro electro mechanical systems, Belfast, 21-25 January 2018

11. Choong YYC, Maleksaeedi S, Eng H, Wei J, Su P-C (2017) 4D printing of high performance shape memory polymer using stereolithography. Mater Des 126:219-225

12. Choong YYC, Maleksaeedi S, Eng H, Su P-C, Wei J (2017) Curing characteristics of shape memory polymers in 3D projection and laser stereolithography. Virtual Phys Prototyp 12:77-84

13. Choi IH, Kim YK, Lee S, Lee SH, Kim J (2015) A pneumatic drop-ondemand printing system with an extended printable liquid range. J Microelectromech Syst 24:768-770

14. Choi M, Humar M, Kim S, Yun S-H (2015) Step-index optical fiber made of biocompatible hydrogels. Adv Mater 27:4081-4086

15. Hao Y, Shih H, Munoz Z, Kemp A, Lin C-C (2014) Visible light cured thiol-vinyl hydrogels with tunable degradation for 3D cell culture. Acta Biomater 10:104-114

16. Park J-W, Shim G-S, Back J-H, Kim H-J, Shin S, Hwang T-S (2016) Characteristic shrinkage evaluation of photocurable materials. Polym Test 56:344-353

17. Abbott JR, Higgins BG (1988) Surface tension of a curing epoxy. J Polym Sci Pol Chem 26:1985-1988

\section{Publisher's Note}

Springer Nature remains neutral with regard to jurisdictional claims in published maps and institutional affiliations. 